\title{
Brain functional connectivity correlates of coping styles
}

\author{
Emiliano Santarnecchi ${ }^{1,2,3,4}$. Giulia Sprugnoli ${ }^{1}$. Elisa Tatti ${ }^{1} \cdot$ Lucia Mencarelli $^{1}$ • Francesco Neri ${ }^{1} \cdot$ Davide Momi $^{1}$. \\ Giorgio Di Lorenzo ${ }^{5,6}$. Alvaro Pascual-Leone ${ }^{2} \cdot$ Simone Rossi ${ }^{1,4,7} \cdot$ Alessandro Rossi ${ }^{1,4}$
}

Published online: 23 March 2018

(C) Psychonomic Society, Inc. 2018

\begin{abstract}
Coping abilities represent the individual set of mental and behavioral strategies adopted when facing stress or traumatic experiences. Coping styles related to avoidance have been linked to a disposition to develop psychiatric disorders such as PTSD, anxiety, and major depression, whereas problem-oriented coping skills have been positively correlated with well-being and high quality of life. Even though coping styles constitute an important determinant of resilience and can impact many aspects of everyday living, no study has investigated their brain functional connectivity underpinnings in humans. Here we analyzed both psychometric scores of coping and resting-state fMRI data from 102 healthy adult participants. Controlling for personality and problem-solving abilities, we identified significant links between the propensity to adopt different coping styles and the functional connectivity profiles of regions belonging to the default mode (DMN) and anterior salience (AS) networks - namely, the anterior cingulate cortex, left frontopolar cortex, and left angular gyrus. Also, a reduced negative correlation between AS and DMN nodes explained variability in one specific coping style, related to avoiding problems while focusing on the emotional component of the stressor at hand, instead of relying on cognitive resources. These results might be integrated with current neurophysiological models of resilience and individual responses to stress, in order to understand the propensity to develop clinical conditions (e.g., PTSD) and predict the outcomes of psychotherapeutic interventions.
\end{abstract}

Keywords Coping $\cdot$ fMRI $\cdot$ Connectivity $\cdot$ Network $\cdot$ Resilience

Emiliano Santarnecchi

esantarn@bidmc.harvard.edu

1 Siena Brain Investigation \& Neuromodulation Laboratory (Si-Bin Lab), Department of Medicine, Surgery and Neuroscience, Neurology and Clinical Neurophysiology Section, University of Siena, Siena, Italy

2 Berenson-Allen Center for Non-Invasive Brain Stimulation, Beth Israel Medical Center, Harvard Medical School, Boston, MA, USA

3 Siena Robotics and Systems Lab (SIRS-Lab), Engineering and Mathematics Department, University of Siena, Siena, Italy

4 Unit of Neurology and Clinical Neurophysiology, Department of Medicine, Surgery and Neuroscience, University of Siena, Siena, Italy

5 Laboratory of Psychophysiology, Department of Systems Medicine, University of Rome Tor Vergata, Rome, Italy

6 Psychiatry and Clinical Psychology Unit, Department of Neurosciences, Fondazione Policlinico Tor Vergata, Rome, Italy

7 Human Physiology Section, Department of Medicine, Surgery and Neuroscience, University of Siena, Siena, Italy
The concept of coping reflects the combination of mental and behavioral strategies implemented in order to master, minimize or tolerate stress and conflict (Lazarus \& Folkman, 1984). Rather than as a specific personality trait or a cognitive ability, coping style is described as a standalone psychological construct, sharing features with constitutional variables such as temperament and personality (e.g., the Big Five personality traits; Caprara, Barbaranelli, Borgogni, \& Perugini, 1993), locus of control (Rotter, 1966), self-efficacy (Bandura, 1994), and extroversion/introversion (H. J. Eysenck \& Eysenck, 1975), as well as with specific skills (e.g., problem solving and executive functions). Given its relevance in determining the development of psychiatric diseases and maintaining physical health, by reducing the health risk of stress (Gleiberman, 2007; Mikulincer \& Solomon, 1989; Wolters Gregório et al., 2015), coping has been extensively studied in the last four decades, as part of an effort to understand how to improve or train specific coping skills for therapeutic purposes (Folkman \& Lazarus, 1988). Coping is also considered a core element of the multidimensional construct of "resilience," which includes interacting factors such as genetics, epigenetics, developmental environment, 
psychosocial factors, cognitive profile, and functional neural circuitry, all contributing to "shield" mind and brain against pathological conditions (Southwick \& Charney, 2012; van der Werff, van den Berg, Pannekoek, Elzinga, \& van der Wee, 2013). For all these reasons, understanding the neurobiology of coping skills constitutes the first step in the implementation of more effective interventions aimed at increasing specific coping styles, via psychotherapy or other methods (Thompson, Arnkoff, \& Glass, 2011; Witek-Janusek et al., 2008).

A variety of definitions of coping have been proposed, and numerous metrics to assess individual coping skills have been developed. Specifically, Folkman and Lazarus $(1980,1985)$ differentiated two major styles of coping: problem-focused and emotion-focused coping. The former reflects active efforts to solve, reconceptualize, or minimize the effects of a stressful situation by focusing on the problem, developing alternative solutions, and acting accordingly. The latter includes strategies that involve self-preoccupation, worry, rumination, or other activities, mostly related to emotion regulation. To study individual coping differences, Folkman and Lazarus (1988) developed a measure called "Ways of Coping," a checklist of problem- and emotion-focused coping strategies that can be applied to a variety of stressful situations. Even though the distinction between problem-focused and emotion-focused coping is an important one, studies have suggested that it might be too simplistic (Folkman \& Lazarus, 1985; Parkes, 1984; Scheier, Weintraub, \& Carver, 1986). For instance, Carver, Scheier, and Weintraub (1989) developed a new measure called the "Coping Orientation to the Problems Experienced" (COPE) inventory, which included 15 coping subscales covering a broad spectrum of stress-management options, ranging from planning to seeking social support and turning to religion. Interestingly, factorial analysis has unveiled a four-factor structure in which the first two factors closely resembled Folkman and Lazarus's $(1980,1985)$ problem-focused and emotion-focused coping. The third factor instead captured a coping style based on seeking social support, to get feedback or advice or to express emotions, and the fourth factor corresponded with attempts to avoid dealing with either the problem or the associated emotions.

Since then, the COPE Inventory had been exported and validated in several countries, including Germany, Italy, France, and Spain. Even though the COPE psychometric core has been replicated for every version, factorial analysis on the datasets collected in different countries has shown how the 15 scales can be assembled in variable latent structures, spanning from three to five factors (Litman, 2006). As expected, both the number and nature of the factors vary depending on culture. For instance, a fifth factor has been identified in the Italian validation of the COPE, reflecting a transcendenceoriented coping style characterized by seeking comfort in mystical and religious practice or thoughts (Sica et al., 2008). However, regardless of the scale employed, research has shown that the prevalence of certain coping styles can predict the predisposition to develop specific clinical conditions. For instance, emotion-focused coping, and particularly avoidance-based coping, is related to a higher incidence of PTSD (e.g., Brousse et al., 2011; Bryant \& Harvey, 1996; Chang et al., 2003; Mikulincer \& Solomon, 1989), whereas problem-focused coping is associated with lower levels of PTSD (Mikulincer \& Solomon, 1989). Moreover, several studies have demonstrated how "inadequate" coping styles, such as emotion-focused and avoidance-oriented ones, are linked to a general criminal lifestyle and also appear to be associated with recidivism in sex offenders (Hanson, Harris, Scott, \& Helmus, 2007). Interestingly, even executive functions (i.e., inhibition, working memory, planning, and flexibility) have been associated with coping style, in both neurological (Goretti et al., 2010; Wolters Gregório et al., 2015) and psychiatric (Lysaker, Clements, Plascak-Hallberg, Knipscheer, \& Wright, 2002) populations. However, very little effort has gone into understanding the neural correlates of coping skills, with only one recent longitudinal study investigating the structural brain correlates of coping (Holz et al., 2016). Specifically, using a self-report questionnaire (the German Stress Coping Questionnaire, SVF78), the authors have highlighted that positive (i.e., problem-oriented) coping styles are associated with increased volume of the anterior cingulate cortex, also showing a link to the development of anxiety and depression disorders.

Apart from Holz et al.'s (2016) correlation with brain volumes, no study has investigated the potential link between coping styles and the human functional connectome (Sporns, 2014). The analysis of spontaneous low-frequency $(<0.1 \mathrm{~Hz})$ fluctuations of the blood oxygen level-dependent (BOLD) signal (Biswal, Yetkin, Haughton, \& Hyde, 1995), recorded using functional magnetic resonance imaging (fMRI), have been established as a reliable and informative approach to probe the healthy brain's functional architecture (Biswal et al., 2010; Sporns, 2013). The analysis of spontaneous interregional connectivity, measured via resting-state functional connectivity (rs-FC), has unveiled functional correlates of cognitive abilities (Santarnecchi, Galli, Polizzotto, Rossi, \& Rossi, 2014; Sporns, 2014; Wang, Song, Jiang, Zhang, \& Yu, 2011), has characterized the signs and symptoms of neurological and psychiatric conditions (Greicius, 2008; Zhang \& Raichle, 2010), and has put forward the idea of even mapping the peculiar features of each individual brain, thus leading to the identification of brain "fingerprints" (Finn et al., 2015). Most importantly, an attempt at identifying the correlates of more subtle and intangible traits such as resilience (Alstott, Breakspear, Hagmann, Cammoun, \& Sporns, 2009; Joyce, Hayasaka, \& Laurienti, 2013; Santarnecchi, Rossi, \& Rossi, 2015) and personality (Adelstein et al., 2011) have been successfully carried out, suggesting the possibility of finding traces of other dimensions, such as coping, as well. 
Given the impact on many aspects of daily life, here we aimed to detect rs-FC patterns explaining individual variability in coping styles. We collected neuroimaging, coping, personality, and problem-solving data in a sample of healthy adult participants. We hypothesized that coping styles will be reflected in the spontaneous activity of brain regions and networks related to executive functioning and planning (to define hierarchy of steps to overcome a given stressful situation), emotion control and inhibition (to suppress automatic avoidance-oriented responses), and internal dialogue and mind wandering (for its role in determining moment-tomoment mentation and evaluation of future and past scenarios). Therefore, among well-known resting-state networks, we hypothesized a major role for (1) the default mode network (DMN; Raichle, 2015); (2) the frontoparietal control network (FPCN), as defined by Dosenbach et al. (2010), or its equivalent executive control network (ECN), by Shirer, Ryali, Rykhlevskaia, Menon, and Greicius (2012); and (3) the anterior salience network (ASN), as defined by Shirer and colleagues, or its equivalent fronto-opercular network (FON), defined by Dosenbach et al.

\section{Method}

\section{Sample}

A total of 102 healthy (age $M \pm S D=27 \pm 9$; 67 females, 35 males; mean education $=17$ years), right-handed (Oldfield Handedness scale; Oldfield, 1971), monolingual native speakers participated to the study. The study was part of a larger multimodal investigation aimed at identifying neurophysiological correlates of cognitive and personality traits, including perturbation-based markers based on noninvasive brain stimulation (NIBS), genetic, electroencephalographic, cognitive, psychometric, and neuroimaging data. Participants were recruited by the University of Siena and Le Scotte Hospital community via flyers and online advertisement. For the present study, the analysis focused on the psychometric and MRI data collected as part of the six data collection visits carried out for the main study. The participants were compensated $€ 20$ for the psychometric assessment and $€ 30$ for the MRI session. The inclusion criteria were (1) 18 to 45 years old, (2) no evidence of neurological or psychiatric disorders, and (3) no counterindications for the acquisition of magnetic resonance imaging (MRI) data (e.g., pregnancy, pacemaker, metal implants). The exclusion criteria were (1) current or past substance abuse, (2) use of a psychotropic medication within three months prior to inclusion, and (3) brain structural abnormalities at the magnetic resonance imaging (MRI) exam. All participants gave their written informed consent to the experimental procedure, which conformed to the Declaration of Helsinki. The study was approved by the local ethics committee.

\section{Data collection}

Participants completed a battery of psychometric tests evaluating their personality profile, coping skills, and problemsolving abilities, as well as other more specific traits such as tendency to dissociation, evaluation of sensation-seeking behavior, eating disorder and obsessive-compulsive behavior. The evaluation took approximately $4 \mathrm{~h}$ to complete. Before or after completion of the psychometric battery, participants participated to a $2 \mathrm{~h}$ long MRI/fMRI session. Both acquisitions took place at the Le Scotte Hospital in Siena (Italy), specifically at the Brain Investigation and Neuromodulation laboratory and at the Neuroradiology department. The present study focused on identifying resting-state connectivity correlates of individual coping skills. Given the link between coping, problem-solving abilities and major personality traits, scores from two of the most validated personality questionnaires (the Big Five Questionnaire [BFQ]: Caprara et al., 1993; and the Eysenck Personality Questionnaire [EPQ]: H. J. Eysenck \& Eysenck, 1975) were also acquired and used as covariates in the neuroimaging analysis. For the same reason, problem-solving abilities were measured via the Problem Solving Inventory (PSI; Heppner, 1988) and used as covariates, along with age and gender. This was intended to provide a net estimate of rs-FC correlates of coping styles, regardless of differences in factors such as extroversion, introversion, openness, and emotional stability. The details about the specific psychometric measures, MRI sequences, and fMRI data processing are reported below.

\section{Coping style assessment}

Coping was evaluated via the COPE-Nuova Versione Italiana (COPE-NVI), an Italian version of the "Coping Orientation to the Problems Experienced" (COPE) validated by Sica et al. (2008) and based on original version inventory by Carver, Scheier, and Weintraub (1989). The COPE-NVI is a self-report questionnaire created to measure different coping strategies. The questionnaire was administered to 458 individuals (50\% females) belonging to the general community of Padova (Italy), together with various measures of psychopathology and a measure of psychological well-being. A confirmatory factor analysis (CFA) identified five independent dimensions, and proved reliable and fairly stable in time. No difference was identified in relation to the level of education, gender, and age (Sica et al., 2008). On average, across the five scales, the questionnaire displayed good test-retest reliability at one month (.81), and an internal consistency (Cronbach's alpha) equals to .83 .

In its final version, the questionnaire consists of 60 items and participants are asked to answer each item using a Likert scale with a score ranging from 1 (I usually do not) to 4 (I usually do it). The questions were created to investigate five coping styles: (1) social support, including items referring to 
the search for sharing opportunities, information and emotional outburst; (2) avoidance strategies ("avoidance-oriented coping" hereafter), including items aimed at investigating denial, behavioural and mental detachment and substance abuse; (3) positive attitude, which evaluates the attitude of acceptance, containment and positive reinterpretation of events; (4) problem-solving ("problem-oriented coping" hereafter), including items aimed at investigating the use of active strategies and planning; and (5) turning to religion, items referred to religion and the absence of humor. Three dimensions of COPE-NVI (avoidance-oriented coping, problem-oriented coping, positive attitude) can be considered specific to coping processes, whereas social support and turning to religion are attitudes and behaviors that affect many aspects of human activity and are not directly associated with psychological well-being. Moreover, avoidance-oriented coping is largely associated with emotional distress, whereas positive attitude and problem-oriented coping are related to less distress and greater psychological well-being.

\section{Personality assessment}

Big Five Questionnaire (BFQ) The BFQ is a test used to evaluate the five fundamental dimensions of human personality. It is based on the "Five-Factor Model of Personality" that has proved to be universal (i.e., valid across countries and social norms) and of great practical relevance for the definition of personality. The Italian version of the BFQ was proposed in 1993 by Caprara et al. (1993). The BFQ identifies five fundamental dimensions describing personality: (1) $\mathrm{E}=$ energy, which is inherent in a confident and enthusiastic orientation toward the various circumstances of life, most of which are interpersonal; (2) $\mathrm{F}=$ friendliness, which includes features like altruism, caring, giving emotional support, but also features such as hostility, indifference to others, selfishness; (3) $\mathrm{C}=$ conscientiousness, which refers to features such as reliability, responsibility and perseverance; (4) $\mathrm{S}=$ emotional stability, which is a very large dimension that includes a variety of features related to anxiety and emotional experience such as mood instability and irritability; and (5) $\mathrm{O}=$ openness, which refers to individual propensity to open up to new ideas and to alternative points of view. Each of the five major dimensions is defined by two subdimensions, describing the opposite boundaries of each dimension: dynamism and dominance (E), cooperativeness and politeness $(\mathrm{F})$, scrupulousness and perseverance $(\mathrm{C})$, emotion control and impulse control (S), openness to culture and experience (O). The BFQ is composed by 132 questions and participants have to answer using a Likert scale with a score ranging from 1 (absolutely false) to 5 (very true). Half the items are positively worded with respect to the scale name, and half are negatively worded in order to control for possible acquiescence response set. The Lie (L) scale is designed to assess the "social desirability" type of response set and measures the tendency to attribute to themselves positive qualities associated with social and intellectual status and the tendency to attribute to themselves morally desirable qualities.

Eysenck Personality Questionnaire (EPQ-R) The EPQ is a selfadministered questionnaire to assess personality. The original version was published in 1975 by Eysenck (H. J. Eysenck \& Eysenck, 1975), and follows the author's model of human personality based on three fundamental dimensions: neuroticism (N), extroversion (E), and psychoticism (P). In the EPQR, the revised version of the EPQ (S. B. G. Eysenck, Eysenck, \& Barrett, 1985), new items were added to the Psychoticism scale. An exploratory factorial analysis of the Italian version of the EPQ-R was performed by Galeazzi, Goti, and Vidotto (1992), and the final version of the questionnaire was published by Dazzi, Pedrabissi, and Santinello (2004). The test is composed by 100 dichotomous (yes/no) items. It is characterized by four scales: (1) "N" scale: neuroticism or emotionality (high score on the $\mathrm{N}$ scale indicate an individual characterized by instability, nervousness and general anxiety); (2) "E" scale: extroversion-introversion (high score on the E scale indicate a person characterized by extroversion, sociability, and impulsiveness); (3) "P" scale: psychoticism or tough-mindedness, also considered a measure of antisocial behavior; and (4) "L" scale: a "lie" scale, to evaluate response biases link to social desirability.

Problem Solving Inventory (PSI) The PSI is a test created to identify and characterize problem-solving style. The PSI is a self-administered questionnaire composed by 35 items based on a 6-point Likert scale (from strongly agree $=1$ to strongly disagree $=6$ ), it was presented for the first time by Heppner (1988) and adapted for Italian population by Mirandola and Soresi (1991). The total score of PSI can be split in three different factors: Problem-Solving Confidence (PSC), Approach-Avoidance Style (AAS), and Personal Control (PC). PSC is measured by 11 items that refer to an individual's belief in one's own problem-solving ability (e.g., "I usually think I can find an effective solution for my problems"); AAS is composed by 16 items that refers to individual approach to problem-solving activities (e.g., "When I have a problem I tend to avoid thinking about it"); PC, composed by five items, refers to individual ability to control one's own emotions while solving problems (e.g., "even though I work on a problem, sometimes I feel like I am mind wandering, and am not getting down to the real issue"). In general, people who perceive themselves as good problem solvers have greater selfefficacy and they feel more in control of their emotional and cognitive abilities. Conversely, people who perceive themselves as bad problem solvers have been shown to be more likely to report depression, suicidal ideation, and anxiety (Heppner, Witty, \& Dixon, 2004; Nezu, 1985). 


\section{MRI data acquisition}

MRI data was acquired on a Philips Intera whole-body scanner. Resting-state fMRI data included 178 volumes with 33 axial slices covering the whole brain, acquired via a $\mathrm{T} 2$ BOLD-sensitive multislice echo-planar imaging (EPI) sequence $(\mathrm{TR} / \mathrm{TE}=2.5 \mathrm{~s} / 32 \mathrm{~ms}$; field of view $=22 \mathrm{~cm}$; image matrix $=64 \times 64$; voxel size $=3.44 \times 3.44 \times 3.8 \mathrm{~mm}^{3}$; flip angle $=75^{\circ}$ ). Structural imaging was performed using a whole brain $\mathrm{T} 1$-weighted fast field echo $1-\mathrm{mm}^{3}$ sequence $(\mathrm{TR} / \mathrm{TE}=$ $30 / 4.6 \mathrm{~ms}$, field of view $=250 \mathrm{~mm}$, matrix $256 \times 256$, flip angle $=30^{\circ}$, slice number $=150$ ). T2-weighted fluid-attenuated inverse recovery images (FLAIR) were also acquired to assess participants white matter integrity. Participants lied in the MRI scanner with their eyes open, while fixating a crosshair in order to avoid excessive eye movements. The MRI technician monitored each participant throughout the entire MRI visit using the camera installed inside the scanner. Particular care was taken to minimize head motion via vacuum cushions and custom-made padding. Participants were provided with earplugs.

\section{fMRI preprocessing}

fMRI data preprocessing and statistical analyses were carried out using SPM8 software (Statistical Parametric Mapping; www.fil.ion.ucl.ac.uk/spm/) and MATLAB 7.5 (MathWorks, MA, USA). The first three volumes of functional images were discarded for each subject to allow for steady-state magnetization. EPI images were slice-time corrected using the interleaved descending acquisition criteria, and realigned and resliced to correct for head motion using a mean functional volume derived from the overall fMRI scans. Participants whose head motion exceeded $1.0 \mathrm{~mm}$ or rotation exceeded 1. $0^{\circ}$ during scanning were excluded. To obtain the better estimation of brain tissues maps, we implemented an optimized segmentation and normalization process using DARTEL (diffeomorphic anatomical registration using exponential Lie algebra) module for SPM8. Briefly, this approach is based on the creation of a customized anatomical template built directly from participants T1-weighted images instead of the canonical one provided with SPM (MNI template, ICBM 152, Montreal Neurological Institute). This allows a finer normalization into standard space and consequently avoids under- or overestimation of brain regions volume possibly induced by the adoption of an external template. Hidden Markov random field model was applied in all segmentation processes in order to remove isolated voxels. Customized tissue prior images and T1weighted template were smoothed using an 8-mm full width at half maximum isotropic Gaussian kernel. Functional images were consequently nonlinearly normalized to standard space and a voxel resampling to (isotropic) $3 \times 3 \times 3 \mathrm{~mm}$ was applied. Linear trends were removed to reduce the influence of the rising temperature of the MRI scanner and all functional volumes were band-pass filtered at $(0.01<f<0.08 \mathrm{~Hz})$ to reduce low-frequency drift. Finally, a CompCor algorithm was applied in order to control physiological high-frequency respiratory and cardiac noise (Behzadi, Restom, Liau, \& Liu, 2007).

\section{Functional connectivity analysis}

Resting-state functional connectivity analysis was implemented using ad-hoc scripts implemented in a Python (Python Software Foundation, https://www.python.org) and MATLAB (The MathWorks, Inc., Natick, Massachusetts, United States) computational environment. To avoid any a priori hypothesis about specific brain regions or networks being correlated with coping styles, the analysis was performed using a two steps procedure. First, the data were analyzed by comparing the connectivity among a set of regions of interest covering cerebral and cerebellar grey matter, including subcortical structures as, for instance, the thalamus and basal ganglia (see the Whole-Brain Analysis section and Fig. 1 $\mathrm{a}^{*}$ ). This provided a set of significant region-to-region connectivities correlated with COPE-NVI scores, also ensuring that results were not due to a priori selection of analysis masks/regions or inflated by the implicit reduction of statistical comparisons. Subsequently, an approach for identifying regions showing more significant connections being correlated with coping was used (network-based statistics [NBS]; Zalesky, Cocchi, Fornito, Murray, \& Bullmore, 2012), identifying the one brain region explaining more variance for each COPE-NVI subscale. NBS takes into account the distribution of significant pairwise connectivities and selects regions that display a higher number of significant connections among the entire set of regions of the anatomical/functional atlas being used, thus reducing spurious results (see Fig. $1 \mathrm{a}^{\wedge}$ ). Indeed, for the five different Coping measures, only three regions were retained as significant after FDR and NBS corrections. The identified regions were then introduced to a seed-based, voxel-wise connectivity analysis (see the Seed-Based Analysis section); specifically, the regions surviving the NBS were used as seeds and their connectivity profiles were correlated with coping scores, identifying clusters of voxels significantly connected with each region of interest. This allowed to look at voxel-level results and further increase spatial specificity. Details about each analysis are reported below.

Whole-brain analysis Functional connectivity (FC) was calculated by computing the Pearson product-moment correlation coefficient between the BOLD time series extracted from each brain region composing the functionally defined atlas by Shen, Tokoglu, Papademetris, and Constable (2013), which include 184 cortical, subcortical, and cerebellar regions of interest (ROIs) sampled at $1-\mathrm{mm}^{3}$ resolution. Separate 
A

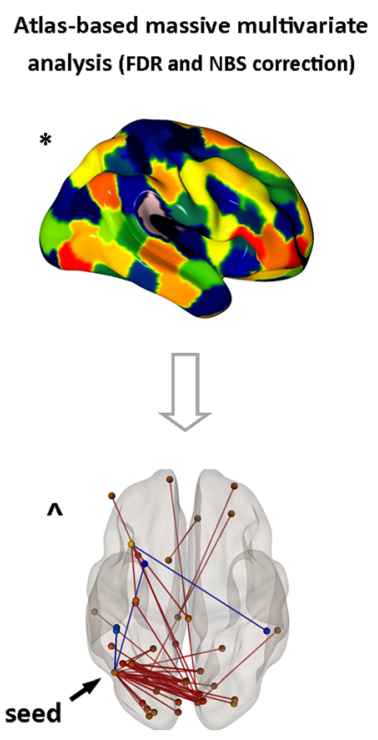

B

Network Labeling via

seed-based connectivity

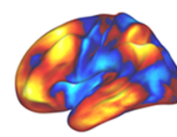

LECN

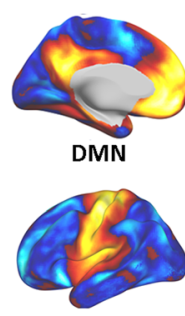

SM

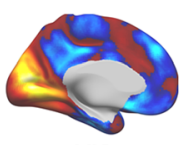

VIS

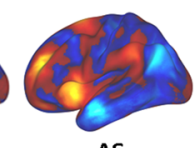

AS
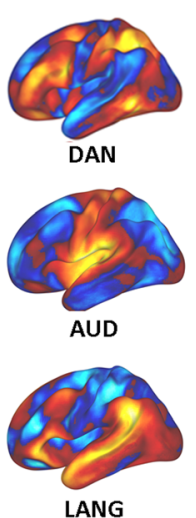

C

Seed-based analysis

(FDR voxel level; FEW cluster level)
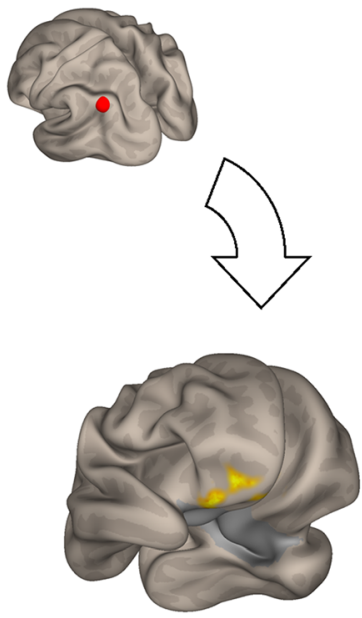

Fig. 1 Functional connectivity analysis. Analysis of the resting-state fMRI data included a first, multivariate analysis based on the functional parcellation atlas $(*)$, leading to the identification of brain regions whose connectivity profiles correlated with COPE scores (panel a). The resulting regions were used as seeds $(\wedge)$ to identify their connectivity profiles and the corresponding resting-state networks (panel $\mathbf{b}$ ), as well as to run a second-level seed-based connectivity analysis to refine the results in panel $\mathbf{a}$ at a higher (i.e., voxel-level) resolution (panel c). $\mathrm{LECN}=$ left executive control network; $\mathrm{AS}=$ anterior salience network; DMN = default mode network; DAN = dorsal attention network; $\mathrm{SM}=$ sensorimotor network; AUD = auditory network; VIS = visual network; LANG = language network; FDR = false discovery rate; FWE = family-wise error regression models were built for each coping scale $(n=5)$, looking at the pairwise connectivity correlates of each style (Fig. 1a). Statistical thresholds of $p>.05$ at the edge (i.e., connection) level and $p>.05$ false discovery correction (FDR) for multiple comparison correction were adopted. The models included age, gender, education, scanning movement parameters, and total brain volume as covariates. Additionally, according to the NBS framework proposed by Zalesky et al. (2012), a second threshold was applied in order to isolate regions of significant changes in connectivity not due to the intrinsic positive manifold usually observed in human rs-FC connectivity matrices (Fig. 1a). The identified regions with high predictive power for coping styles were then used as seed regions to determine their FC profiles (see the next section). These profiles were also visually inspected and associated with well-known resting-state networks, to ease interpretation of the results (Fig. 1b).

Seed-based analysis To increase the spatial resolution of the analysis, the seed regions identified via NBS were introduced into a second regression model to predict coping scores by looking at seed-to-voxel patterns (Fig. $1 \mathrm{c} ; p>.05$ at voxel level and family-wise error, FWE, correction for cluster size correction).

All the analyses included age, gender, education, scanning movement parameters, and total brain volume as covariates. Moreover, in an effort to provide estimates of FC correlates of coping that were not dependent on — or just reflect - trait-level personality dimensions, individual scores on the BFQ, EPQ, and PSI were included as covariates in the analysis.

\section{Results}

\section{Psychometric assessment}

The results of the personality tests are displayed in Fig. 2. In line with what was previously described in studies of healthy participants (Caprara et al., 1993; Galeazzi et al., 1992; Sica et al., 2008), our sample reported, as compared to the distribution of scores at the various scales, lower scores for neuroticism on the BFQ, lower psychoticism on the EPQ, and lower scores on the Transcendence and Avoidance coping scales of the COPE. As for the PSI, participants displayed higher scores on the Approach/Avoidance Problem-Solving scale, meaning that they tended to engage more often in problem-oriented than in avoidance behaviors, suggesting high similarity with COPE-NVI scores.

\section{Whole-brain connectivity analysis}

A mass multivariate connectivity analysis identified significant associations for three out of the five coping styles: namely, avoidance-oriented $[F(2,13)=4.23, p<.006$, Cohen's $d=$ 


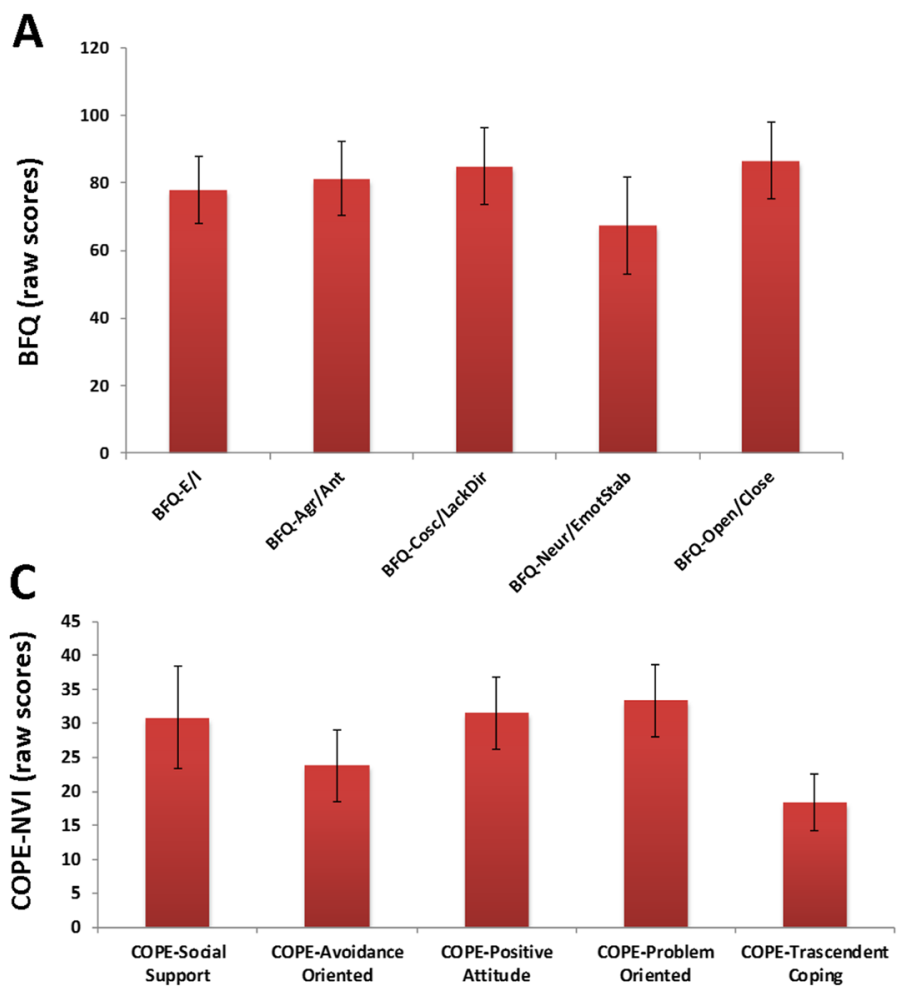

Fig. 2 Psychometric scores. The mean and standard deviation are shown for each personality, coping, and problem-solving measure. $\mathrm{BFQ}=\mathrm{Big}$ Five Questionnaire; E/I = Extroversion/Introversion; Agr $/$ Ant = Agreeability/Antagonism; Cosc/LackDir $=$ Conscientiousness $/$ Lack of

$0.341]$, problem-oriented $[F(2,13)=3.68, p<.003$, Cohen's $d$ $=0.311]$, and social-support-oriented $[F(2,13)=3.29, p<.01$, Cohen's $d=0.296$ ] coping (Fig. 3). No significant predictors were identified for the transcendence coping $(p>.38)$ and positive attitude $(p>.12)$ subscales. Specifically, avoidanceoriented coping correlates with the connectivity profile of the anterior cingulate cortex (ACC) as captured by ROI 36 of the Shen atlas (BA32/6), which mapped on the AS network (Fig. $3 b)$. Problem-oriented coping was related to spontaneous connectivity of the left angular gyrus (ROI 159, BA39) and mapped on the DMN (Fig. 3b). Social-support-oriented coping was related to the activity of the left frontopolar cortex (ROI 178, BA10) and mapped on the DMN (Fig. 3b).

\section{Seed-based analysis}

The significant regions highlighted in the whole-brain analysis (ACC, left angular gyrus, left frontopolar cortex) were used as ROIs in a seed-based FC analysis. ACC displayed a negative correlation between avoidance-oriented coping scores and connectivity with two significant clusters, located in the medial prefrontal and precuneus cortices bilaterally (Fig. 3c) (for statistical results and the MNI coordinates of each cluster, see Table 1). The connectivity of the left angular gyrus displayed a positive correlation with problem-oriented coping scores, with a significant cluster located in the visual cortex bilaterally

\section{B}
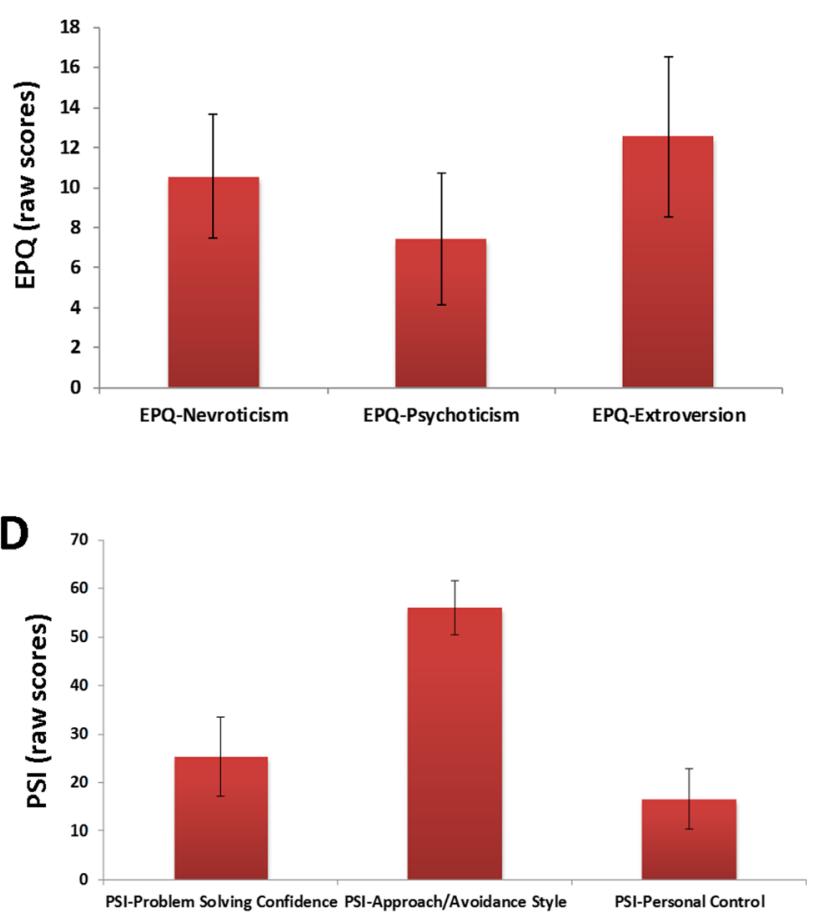

Direction; Neur/EmotStab $=$ Neuroticism/Emotional Stability; Open/ Close = Openness $/$ Closedness; EPQ = Eysenck Personality Questionnaire; PSI $=$ Problem Solving Inventory

(Table 1 and Fig. 3c). The connectivity of the left frontopolar cortex displayed a negative correlation with social-supportoriented coping scores, with a significant cluster located in the right temporal pole region (Table 1 and Fig. 3c).

Given the nature of brain connectivity patterns, which can reflect both positive and negative connectivity among brain regions' activity, a positive or negative association with a cognitive/behavioral variable might result in findings difficult to interpret. For instance, higher intelligence can be correlated with decreased connectivity between two brain regions but, in the case of two regions showing negative spontaneous connectivity at rest, this actually means that higher intelligence reflects a decrease in their "desynchronization," and possibly a hint of a shift toward positive connectivity. Therefore, to ease interpretation of the results, significant correlations between each seed region's connectivity and the corresponding coping scores have been plotted in Fig. 4. In the case of ACC's connectivity and avoidance-oriented coping, the scatterplots in Fig. 4a suggest that more negative correlations between $\mathrm{ACC}$ and the DMN correspond to higher avoidance-related coping (i.e., more negative values on the $y$-axis, reporting ASDMN connectivity, correspond to more positive values on the $x$-axis, representing coping scores). The same interpretation applies to the other significant correlations, except for problem-oriented coping, which shows an opposite pattern (i.e., the more positive the correlation between left angular 


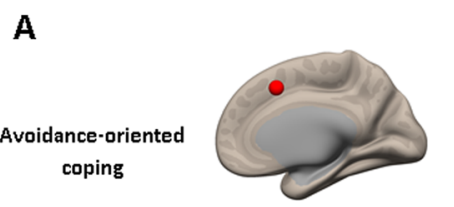

Anterior Cingulate Cortex (BA32/6)
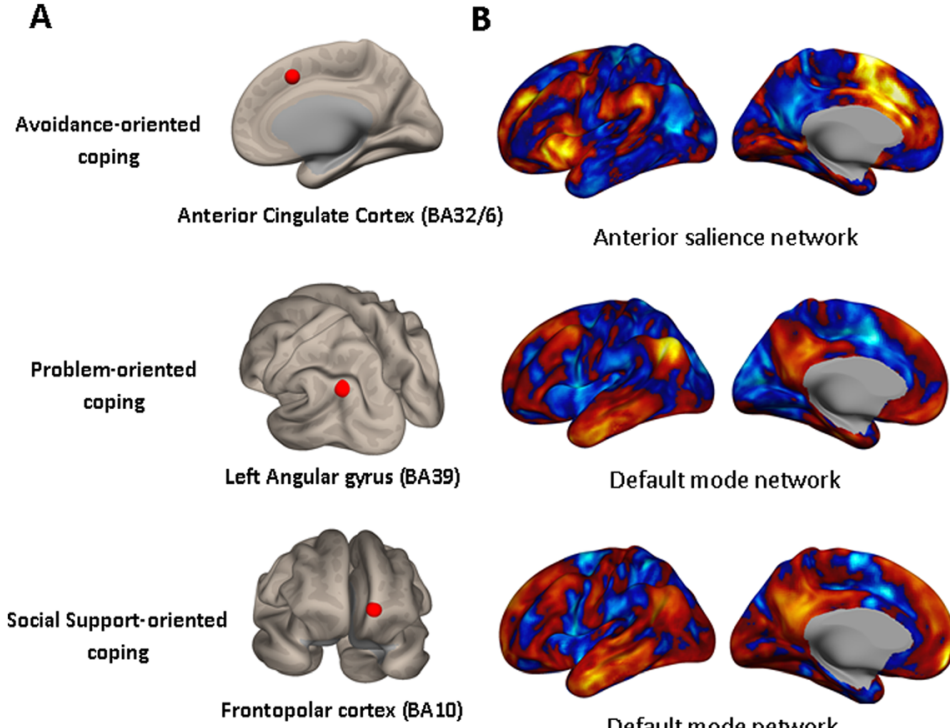

Frontopolar cortex (BA10)
Anterior salience network
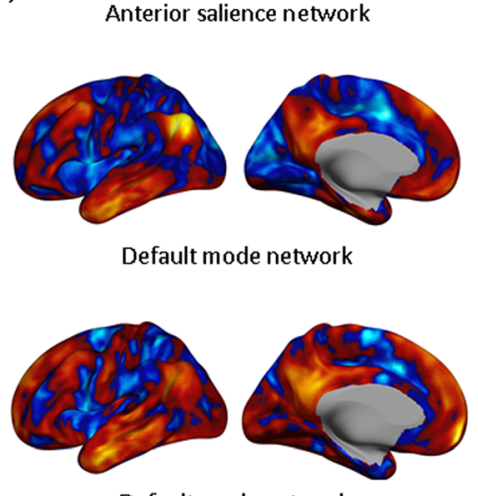

Default mode network

Default mode network
C

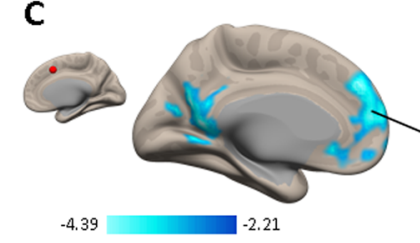

D

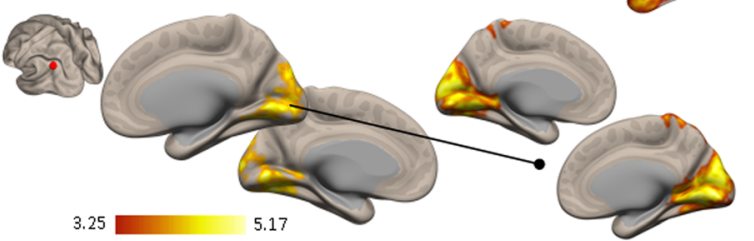

3.25

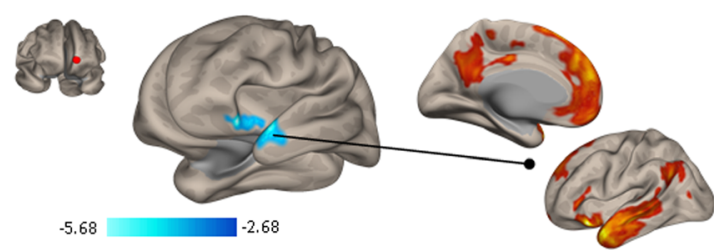

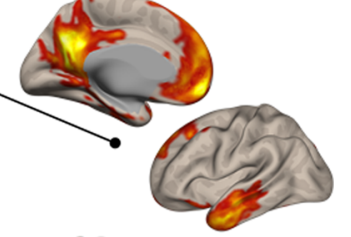

Fig. 3 Pairwise and seed-based connectivity correlates of coping styles. The anatomical locations of regions displaying a significant association with coping styles are reported in panel a, along with their seed-based connectivity profiles (panel b), which highlighted two main resting-state networks. The regions in panel a were used as seeds to explore their seedto-brain correlations in relation to individual coping scores (panel C). The results in panel a were obtained using a $p<.05 \mathrm{FDR}$ and NBS. The results

gyrus [DMN] and visual cortex, the higher the problemoriented coping scores). A more detailed examination of the results is included as part of the discussion section below.

\section{Discussion}

We investigated whether individual coping styles might correlate with spontaneous brain activity in humans. By analyzing psychometric and fMRI data of 102 healthy participants, we identified significant links between propensity to adopt different coping styles in stressful situations and functional connectivity profile of three brain regions belonging to the default mode (Raichle, 2015) and anterior salience network (Uddin, 2014). The interplay between nodes of the two networks also explain variability in one specific coping style related to avoidance, suggesting the AS and DMN as key networks for explaining individual differences in coping style. These results could be integrated with current neurophysiological models of resilience and response to stress, in order to identify potential mechanisms for coping enhancement in therapeutic settings.

\section{Resting-state brain connectivity and coping}

In general, the functional connectivity correlates of coping skills point to the activity of regions belonging to two main resting-state networks - that is, AS and DMN. Especially for in panel $\mathbf{c}$ were obtained by applying a $p<.05$ FDR correction at the voxel level and FWE correction at the cluster level. To identify the corresponding resting-state networks, the results in panel $\mathrm{C}$ were also analyzed via seed-based analysis (panel $\mathbf{d}$ ), again highlighting the widespread load on structures resembling the default mode network. FDR = false discovery rate; NBS = network-based statistics; FWE = family-wise error

the latter, its nodes are correlated with problem-oriented (left angular gyrus) and social support-oriented coping (left frontopolar cortex), whereas the region correlated with avoidance-oriented coping (ACC) also explains individual variability in this coping style by means of its negative correlation with medial structures of the DMN. Even though coping styles reflect the manifestation of behavior on a relatively long time scale (i.e., days, weeks, months), one could hypothesize that the individual propensity to express one style or another is presumably linked to spontaneous brain patterns guiding the "automatic" selection of one stress-related response instead of another. In this context, relationships of coping to the activity of two resting-state networks, linked to e.g. emotional processing, viscero-somatic perception, the integration of somatic signals for interoceptive awareness, the determination of stimulus salience and attentional focus (AS; Chiong et al., 2013; Uddin, 2014), as well as imagination of past and future scenarios, mind wandering, and autobiographical memory (DMN; Harrison et al., 2008; Raichle, 2015), seem plausible. Indeed, a recently published study on the brain structural correlates of coping found a strong correlation with the volume of the ACC, one of the region also identified in the present study (Holz et al., 2016). Moreover, a study investigating the functional connectivity fMRI correlates of psychological resilience, a construct that also relates to coping abilities, have recently demonstrated a link with activity of the AS network (Kong, Wang, Hu, \& Liu, 2015). The role of the AS in determine the salience of incoming stimuli, and therefore 
Table 1 Seed-based connectivity results

\begin{tabular}{|c|c|c|c|c|c|c|c|c|c|}
\hline \multirow[t]{2}{*}{ Coping Scale } & \multicolumn{3}{|l|}{ MNI } & \multirow[t]{2}{*}{ Size (voxels) } & \multirow[t]{2}{*}{ Peak $p$-FDR } & \multirow[t]{2}{*}{ Cluster $p$-FWE } & \multicolumn{3}{|c|}{ Location/Label } \\
\hline & $x$ & $y$ & $z$ & & & & Voxels & Hemisphere & Area \\
\hline \multicolumn{10}{|c|}{ Avoidance-oriented coping } \\
\hline & \multirow[t]{3}{*}{-12} & \multirow[t]{3}{*}{-68} & \multirow[t]{3}{*}{22} & \multirow[t]{3}{*}{2,287} & \multirow[t]{3}{*}{.002} & \multirow[t]{3}{*}{.01} & 1,532 & RL & Precuneus \\
\hline & & & & & & & 294 & RL & Cingulate gyrus \\
\hline & & & & & & & 106 & $\mathrm{~L}$ & Lingual gyrus \\
\hline & \multirow[t]{5}{*}{4} & \multirow[t]{5}{*}{68} & \multirow{5}{*}{-12} & \multirow[t]{5}{*}{1,055} & \multirow[t]{5}{*}{.008} & \multirow[t]{5}{*}{.02} & 276 & $\mathrm{~L}$ & Frontal pole \\
\hline & & & & & & & 168 & $\mathrm{~L}$ & Superior frontal gyrus \\
\hline & & & & & & & 140 & $\mathrm{R}$ & Frontal pole \\
\hline & & & & & & & 120 & $\mathrm{~L}$ & Paracingulate gyrus \\
\hline & & & & & & & 100 & $\mathrm{R}$ & Superior frontal gyrus \\
\hline \multicolumn{10}{|c|}{ Problem-oriented coping } \\
\hline & \multirow[t]{9}{*}{-16} & \multirow[t]{9}{*}{-74} & \multirow[t]{9}{*}{-10} & \multirow[t]{9}{*}{5,337} & \multirow[t]{9}{*}{.006} & \multirow[t]{9}{*}{.02} & 729 & $\mathrm{R}$ & Occipital pole \\
\hline & & & & & & & 689 & $\mathrm{~L}$ & Lingual gyrus \\
\hline & & & & & & & 623 & $\mathrm{R}$ & Lingual gyrus \\
\hline & & & & & & & 546 & $\mathrm{~L}$ & Occipital pole \\
\hline & & & & & & & 468 & $\mathrm{~L}$ & Fusiform gyrus \\
\hline & & & & & & & 305 & $\mathrm{R}$ & Intracalcarine cortex \\
\hline & & & & & & & 217 & $\mathrm{R}$ & Cuneus \\
\hline & & & & & & & 126 & $\mathrm{~L}$ & Intracalcarine cortex \\
\hline & & & & & & & 106 & $\mathrm{~L}$ & Fusiform cortex \\
\hline \multicolumn{10}{|c|}{ Social-support coping } \\
\hline & \multirow[t]{2}{*}{-36} & \multirow[t]{2}{*}{20} & \multirow[t]{2}{*}{-22} & \multirow[t]{2}{*}{542} & \multirow[t]{2}{*}{.009} & .02 & 276 & $\mathrm{~L}$ & Temporal pole \\
\hline & & & & & & & 143 & $\mathrm{~L}$ & Frontoorbital cortex \\
\hline
\end{tabular}

The Montreal Neurological Institute (MNI) coordinates, size, and anatomical labeling of significant clusters identified in the seed-based analysis (see Fig. 3c) are displayed. The analysis is based on the seed regions resulting from the multivariate atlas-based analysis displayed in Fig. 3a

the consequent engagement of different other resting-state networks for processing such information, has been recently postulated (Goulden et al., 2014). According to this view, the AS triggers the activation of either the DMN or the executive control network (ECN) - and corresponding deactivation of the other one-depending on the type of cognitive states at hand: autobiographical memory, prospection, theory of mind, navigation, and 'personal' moral reasoning activate the DMN, whereas tasks require a non-moral or impersonal dilemma activate the ECN (Harrison et al., 2008; Spreng \& Schacter, 2012). Interestingly, the relevance of $\mathrm{AS} \longleftrightarrow \longrightarrow \mathrm{DMN}$ interaction has been also stressed in other contexts related to cognitive reserve, neurodegenerative disorders and aging. For instance, the magnitude of AS-DMN negative correlation in patients with frontotemporal dementia (FTD) and Alzheimer's disease $(\mathrm{AD})$ have been significantly related to the severity of patients' cognitive decline, while also displaying a differential trajectory of alteration (i.e., increased in FTD, decreased in AD; Zhou et al., 2010). Moreover, the strength of the negative correlation between the DMN and the rest of the brain (in particular the AS and the dorsal attention network, DAN) has been linked to cognitive performance in healthy adults (Spreng et al., 2013) and it shows a declining trajectory even in healthy aging (Spreng, Stevens, Viviano, \& Schacter, 2016). In the context of coping styles, AS-DMN dynamics might constitute a framework to understand the minute-to-minute decisions reflecting individual coping styles, and their impact on a larger timescale like the one captured by a trait-level coping questionnaire.

The tendency to express more avoidance- or problem-oriented coping strategies might reflect the spontaneous "filtering" process played by the AS network. In the present study, this is reflected by the involvement of ACC, a structure associated to a wide range of emotional and cognitive functions such as flexibility, working memory, inhibition, motivation and, most importantly, emotion regulation (Bush, 2000). Notably, the concept of coping and resilience has been previously associated with flexible emotional responsiveness and flexible control in processing affective and nonaffective material (Genet \& Siemer, 2011; Waugh, Thompson, \& Gotlib, 2011). Furthermore, modifications of the spontaneous activity of ACC have been reported in multiple psychiatric conditions under which poor coping and resilience skills have been also documented, such as major depression disorder (Horn et al., 
A
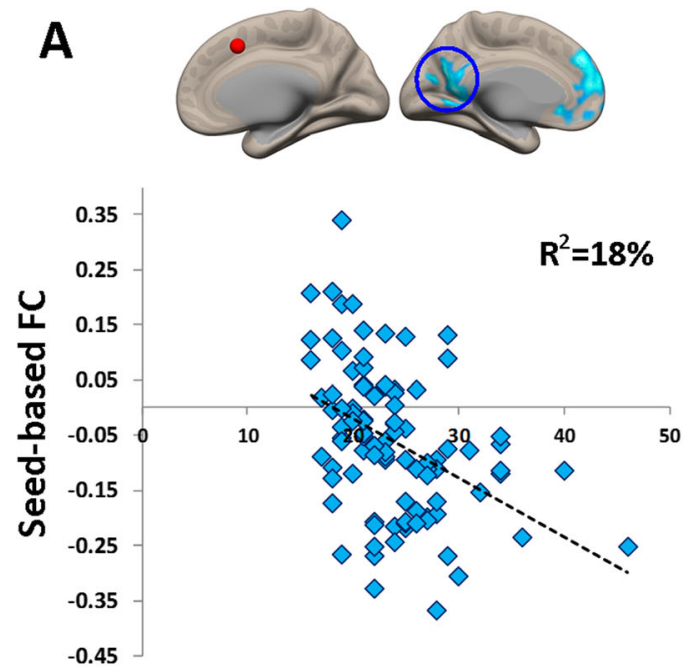

Avoidance-oriented coping

B
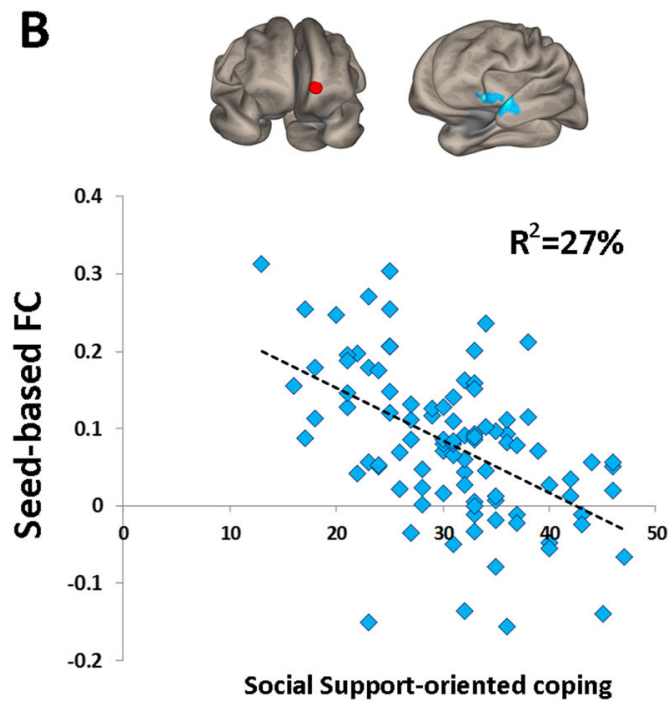

Fig. 4 Correlations between seed-based connectivity and coping scores. The individual scores supporting the patterns of connectivity-coping correlations are displayed in Fig. 2, shown here for (a) avoidance-oriented

2010; Sprengelmeyer et al., 2011; van Tol et al., 2010), PTSD (Karl et al., 2006), and anxiety disorders (van Tol et al., 2010). The same seems to apply to the AS in general, with evidence of its alterations in anxiety disorders (mainly PTSD), generalized anxiety disorder, and social anxiety disorder (Peterson, Thome, Frewen, \& Lanius, 2014). Therefore, the link between ACC connectivity and coping styles highlighted in the present study might point to a dual role of ACC expressed through both its pivotal role during emotion-regulation processes as well as its role in executive functioning, especially in relation to inhibition (Albert, López-Martín, Tapia, Montoya, \& Carretié, 2012). Future studies should investigate the potential modulatory effect of executive functions over the link between coping and ACC connectivity profile.
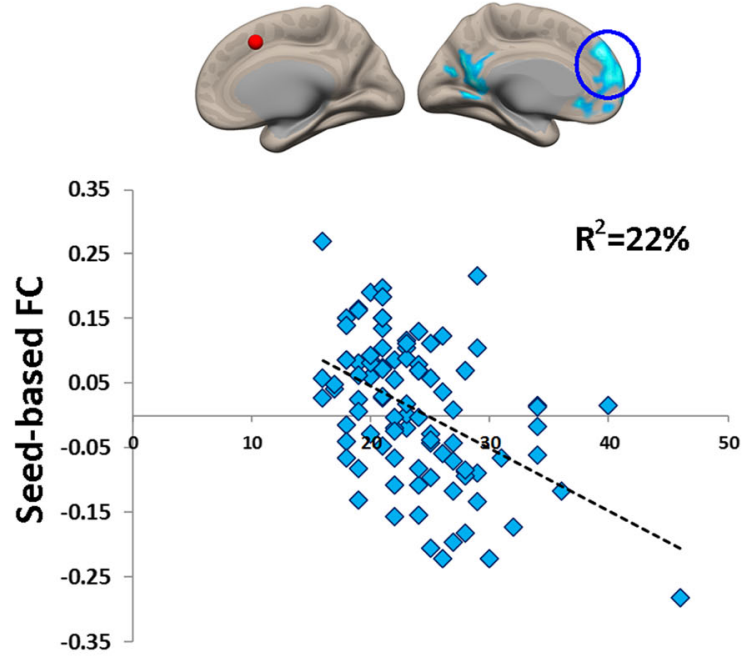

Avoidance-oriented coping
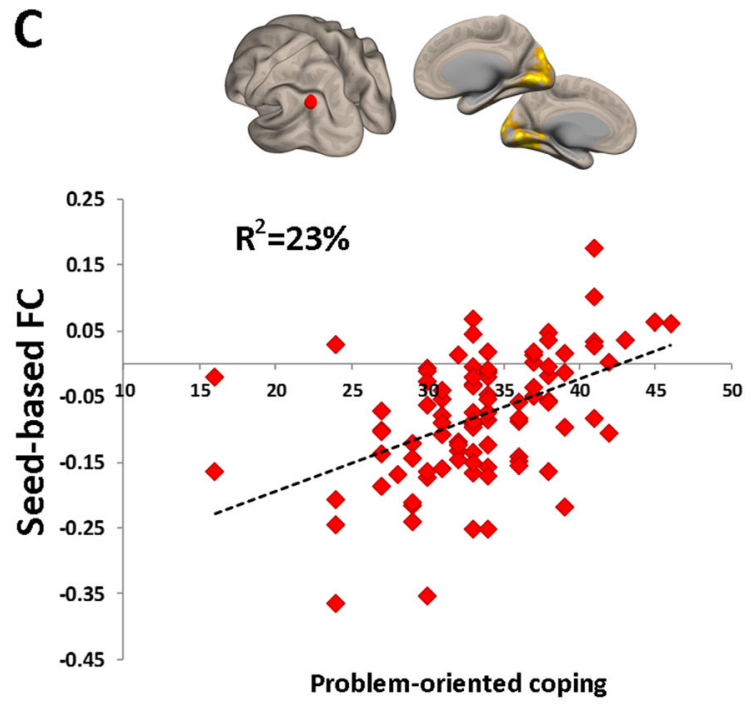

coping, (b) problem-oriented coping, and (c) social-support-oriented coping. $\mathrm{FC}=$ functional connectivity; $R^{2}=$ percentage of variance in coping scores explained by the $\mathrm{FC}$ data

The role played by the specific connectivity patterns highlighted for problem-oriented coping style is less clear. Increase in connectivity between left angular and bilateral visual cortices explain $23 \%$ of behavioral difference in problem-oriented coping. However, analysis of individual scores and $\mathrm{FC}$ values suggests that, instead of an increase in positive connectivity, higher coping scores are supported by a reduction of the physiological negative correlation between angular and visual cortex, with highest scores obtained by participants with a connectivity magnitude around $r=0$ (i.e., no connectivity). If interpreted as a shift toward a null connectivity between DMN and visual areas, this might represent a segregation between internal, mind-wandering-related processes linked to reevaluating ongoing personal life dynamics (and possible 
coping strategies) and external visual stimuli. Instead, a pattern of weak but positive correlation between the two systems has been documented in participants undergoing a stressful social task (Clemens et al., 2017). In both cases, the pattern of correlation requires more data to be fully understood.

As for social-support coping, decrease of positive connectivity between the left frontopolar cortex and temporal pole structures corresponds to higher levels of seeking for social support. As we observed for problem-oriented coping, this goes in the direction of a reversal of physiological restingstate network patterns. Frontopolar and temporal pole are indeed positively correlated structures of the DMN, whose local and synchronized activity has been related to theory of mind (Carrington \& Bailey, 2009) and the processing of social interactions ( $\mathrm{Pu}$ et al., 2016), with a focus on social rejection (Lee et al., 2014). Both of these elements might play roles when one considers seeking support. Additional investigations will be needed to fully understand the nature of this within-network decrease in synchronicity/connectivity. More generally, coping strategies based on social support definitely require more attention, given that the link between the presence of social support - and the behavior of actively seeking social support - has been associated with psychological hardiness and flourishing in the face of adverse life events (Ozbay, Fitterling, Charney, \& Southwick, 2008), whereas the inverse (i.e., poorer social support) has been linked to psychiatric disorders including PTSD (Tsai, Harpaz-Rotem, Pietrzak, \& Southwick, 2012). The tendency to search for social support might be a crucial determinant of psychological well-being, as much as the ability to cognitively cope with stressors, and these forms of coping could potentially share neural substrates (Kong, Hu, Wang, Song, \& Liu, 2015; Kong, Xue, \& Wang, 2016).

Notably, all the correlations highlighted in the present study were obtained after controlling for individual differences in personality traits, according to the two major personality theories (i.e., five- and three-factor personality decomposition). The present results suggest that the link between spontaneous brain connectivity and coping styles is not driven by general personality structure, suggesting the idea of copingspecific neurophysiological dynamics. However, recent evidence has suggested the need for longitudinal assessment of personality measures to account for intrinsic "statedependent" noise in personality assessment (Costantini et al., 2017). Future investigations should include multiple personality data points, thus enabling network-based analysis of both fMRI and psychometric data.

\section{Understand coping to promote resilience}

The possibilities to increase coping abilities and resilience are appealing, with potentially transformative impacts in psychotherapeutic settings. Several enhancement approaches have been investigated, including mindfulness training (Thompson et al., 2011), controlled stress exposure (i.e., stress inoculation training; Craske et al., 2008; McNally, 2007; Tryon, 2005), and behavioral training targeting psychosocial risk factors (Karatsoreos \& McEwen, 2011). Although these interventions might induce psychological and cognitive effects, their nature as behavioral interventions does not target the specific brain circuitry responsible for individual differences in coping/resilience, like the networks potentially identified in the present study. Interestingly, the possibility of using noninvasive brain stimulation (NIBS) to modulate- - excite or inhibit - the activity of specific brain regions or entire networks constitutes an appealing scenario in both healthy and pathological brains (e.g., using transcranial direct current stimulation or transcranial magnetic stimulation; Liew, Santarnecchi, Buch, \& Cohen, 2014; Tatti, Rossi, Innocenti, Rossi, \& Santarnecchi, 2016), with potential applications for the causal investigation of brain-function dualism (PascualLeone, Bartres-Faz, \& Keenan, 1999; Pascual-Leone \& Pridmore, 1995), as well as for cognitive enhancement (Polania, Nitsche, Korman, Batsikadze, \& Paulus, 2012; Santarnecchi et al., 2016; Santarnecchi et al., 2013; Snowball et al., 2013). If the results of the present investigation - pointing to a pivotal role for the activity of the default mode and anterior salience networks - are replicated, NIBS could be used to synchronize/desynchronize such networks (e.g., Polania et al., 2012), perhaps also in combination with the aforementioned behavioral interventions.

Moreover, the concept of coping has similarities with evolutionary biology and neurophysiology models postulating that resilience is an intrinsic property of complex systems, providing the system at hand (e.g., the human brain) with greater abilities to overcome loss of integrity and face external stressors (Kitano, 2004). Interestingly, although the neurophysiological underpinnings of brain resilience have been mapped using neuroimaging techniques (Alstott et al., 2009; Joyce et al., 2013) and also related to cognitive abilities (Santarnecchi et al., 2015), no studies have tested the link between brain resilience to external attacks (in the form of simulated lesions) and coping styles. The identification of brain regions, or specific connectivity patterns, supporting resilience and positive coping styles could help define targets for NIBS and allow for causal investigation of the potential neuroanatomical link between PTSD, vulnerability, and stress (McEwen, Gray, \& Nasca, 2015; van der Werff et al., 2013).

\section{Conclusion}

Individual coping styles show a link with the spontaneous interplay of regions - and their associated networks - related to mind wandering, planning, emotion regulation, and inhibition. The identification of brain-based targets for coping 
processes might help identifying effective strategies for their modulation, with a potential benefit for both the prevention and treatment of psychological distress and psychiatric conditions.

Author note All authors report no conflicts of interest.

\section{References}

Adelstein, J. S., Shehzad, Z., Mennes, M., Deyoung, C. G., Zuo, X. N., Kelly, C., ... Milham, M. P. (2011). Personality is reflected in the brain's intrinsic functional architecture. PLOS ONE, 6, e27633. https://doi.org/10.1371/journal.pone.0027633

Albert, J., López-Martín, S., Tapia, M., Montoya, D., \& Carretié, L. (2012). The role of the anterior cingulate cortex in emotional response inhibition. Human Brain Mapping, 33, 2147-2160. https:// doi.org $/ 10.1002 / \mathrm{hbm} .21347$

Alstott, J., Breakspear, M., Hagmann, P., Cammoun, L., \& Sporns, O. (2009). Modeling the impact of lesions in the human brain. PLoS Computational Biology, 5, e1000408.

Bandura, A. (1994). Self-efficacy. In Encyclopedia of human behavior (Vol. 4, pp. 71-81). New York, NY: Academic Press.

Behzadi, Y., Restom, K., Liau, J., \& Liu, T. T. (2007). A component based noise correction method (CompCor) for BOLD and perfusion based fMRI. NeuroImage, 37, 90-101.

Biswal, B., Yetkin, F. Z., Haughton, V. M., \& Hyde, J. S. (1995). Functional connectivity in the motor cortex of resting human brain using echo-planar MRI. Magnetic Resonance, 34, 537-541.

Biswal, B. B., Mennes, M., Zuo, X. N., Gohel, S., Kelly, C., Smith, S. M., ... Milham, M. P. (2010). Toward discovery science of human brain function. Proceedings of the National Academy of Sciences, 107, 4734 4739. https://doi.org/10.1073/pnas.0911855107

Brousse, G., Arnaud, B., Roger, J. D., Geneste, J., Bourguet, D., Zaplana F., ... Jehel L. (2011). Management of traumatic events: Influence of emotion-centered coping strategies on the occurrence of dissociation and post-traumatic stress disorder. Neuropsychiatric Disease and Treatment, 7, 127-133.

Bryant, R. A., \& Harvey, A. G. (1996). Initial posttraumatic stress responses following motor vehicle accidents. Journal of Traumatic Stress, 9, 223-234.

Bush, G. (2000). Cognitive and emotional influences in anterior cingulate cortex. Trends in Cognitive Sciences, 4, 215-222. Retrieved August 12, 2017, from www.ncbi.nlm.nih.gov/ pubmed/10827444

Caprara, G. V., Barbaranelli, C., Borgogni, L., \& Perugini, M. (1993). The "Big Five Questionnaire": A new questionnaire to assess the five factor model. Personality and Individual Differences, 15, 281-288.

Carrington, S. J., \& Bailey, A. J. (2009). Are there theory of mind regions in the brain? A review of the neuroimaging literature. Human Brain Mapping, 30, 2313-2335. https://doi.org/10.1002/hbm.20671

Carver, C. S., Scheier, M. F., \& Weintraub, J. K. (1989). Assessing coping strategies: A theoretically based approach. Journal of Personality and Social Psychology, 56, 267-283.

Chang, C.-M., Lee, L.-C., Connor, K. M., Davidson, J. R. T., Jeffries, K., \& Lai, T.-J. (2003). Posttraumatic distress and coping strategies among rescue workers after an earthquake. Journal of Nervous and Mental Disorders, 191, 391-398.

Chiong, W., Wilson, S. M., D'Esposito, M., Kayser, A. S., Grossman, S. N., Poorzand, P., ... Rankin, K. P. (2013). The salience network causally influences default mode network activity during moral reasoning. Brain, 136, 1929-1941. https://doi.org/10.1093/brain/ awt066
Clemens, B., Wagels, L., Bauchmüller, M., Bergs, R., Habel, U., \& Kohn, N. (2017). Alerted default mode: Functional connectivity changes in the aftermath of social stress. Scientific Reports, 7, 40180.

Costantini, G., Richetin, J., Preti, E., Casini, E., Epskamp, S., \& Perugini, M. (2017). Stability and variability of personality networks: A tutorial on recent developments in network psychometrics. Personality and Individual Differences, 12, e0186695. https://doi.org/10.1371/ journal.pone.0186695

Craske, M. G., Kircanski, K., Zelikowsky, M., Mystkowski, J., Chowdhury, N., \& Baker, A. (2008). Optimizing inhibitory learning during exposure therapy. Behaviour Research and Therapy, 46, 5-27.

Dazzi, C., Pedrabissi, L., \& Santinello, M. (2004). Adattamento italiano delle Scale di Personalità Eysenck per adulti. Organizzazioni Speciali O.S. Retrieved August 1, 2017, from hdl.handle.net/ $11577 / 2429135$

Dosenbach, N. U., Nardos, B., Cohen, A. L., Fair, D. A., Power, J. D., Church, J. A., ... Schlaggar, B. L. (2010). Prediction of individual brain maturity using fMRI. Science, 329, 1358-1361.

Eysenck, H. J.., \& Eysenck, S. B. G. (1975). Manual of the Eysenck Personality Questionnaire. London, UK: Hodder \& Stoughton.

Eysenck, S. B. G., Eysenck, H. J., \& Barrett, P. (1985). A revised version of the psychoticism scale. Personality and Individual Differences, 6 , 21-29.

Finn, E. S., Shen, X., Scheinost, D., Rosenberg, M. D., Huang, J., Chun, M. M., ... Constable, R. T. (2015). Functional connectome fingerprinting: Identifying individuals using patterns of brain connectivity. Nature Neuroscience, 18, 1664-1671.

Folkman, S., \& Lazarus, R. S. (1980). An analysis of coping in a middleaged community sample. Journal of Health and Social Behavior, 21, 219-239.

Folkman, S., \& Lazarus, R. S. (1985). If it changes it must be a process: Study of emotion and coping during three stages of a college examination. Journal of Personality and Social Psychology, 48, 150-170.

Folkman, S., \& Lazarus, R. S. (1988). Coping as a mediator of emotion. Journal of Personality and Social Psychology, 54, 466-475.

Galeazzi, A., Goti, F., \& Vidotto, G. (1992). Le componenti dell'Eysenck Personality Questionnaire-Revised (EPQ-R). Bolletino di Psicologia Applicata, 202, 13-21.

Genet, J. J., \& Siemer, M. (2011). Flexible control in processing affective and non-affective material predicts individual differences in trait resilience. Cognition and Emotion, 25, 380-388.

Gleiberman, L. (2007). Repressive/defensive coping, blood pressure, and cardiovascular rehabilitation. Current Hypertension Reports, 9, 7 12. Retrieved July 30, 2017, from https:/www.ncbi.nlm.nih.gov/ pubmed/17362665

Goretti, B., Portaccio, E., Zipoli, V., Hakiki, B., Siracusa, G., Sorbi, S., \& Amato, M. P. (2010). Impact of cognitive impairment on coping strategies in multiple sclerosis. Clinical Neurology and Neurosurgery, 112, 127-130.

Goulden, N., Khusnulina, A., Davis, N. J., Bracewell, R. M., Bokde, A. L., McNulty, J. P., \& Mullins, P. G. (2014). The salience network is responsible for switching between the default mode network and the central executive network: Replication from DCM. NeuroImage, 99, 180-190.

Greicius, M. (2008). Resting-state functional connectivity in neuropsychiatric disorders. Current Opinion in Neurology, 21, 424-430. https://doi.org/10.1097/WCO.0b013e328306f2c5

Hanson, R. K., Harris, A. J. R., Scott, T.-L., \& Helmus, L. (2007). Assessing the risk of sexual offenders on community supervision: The Dynamic Supervision Project (Technical Report 2007-05). Ottawa, ON: Public Safety Canada. Retrieved August 6, 2017, from https://www.publicsafety.gc.ca/cnt/rsrcs/pblctns/ssssng-rsk-sxlffndrs/index-en.aspx

Harrison, B. J., Pujol, J., Lopez-Sola, M., Hernandez-Ribas, R., Deus, J., Ortiz, H., ... Cardoner, N. (2008). Consistency and functional specialization in the default mode brain network. Proceedings of the National Academy of Sciences, 105, 9781-9786. 
Heppner, P. (1988). The problem solving inventory. Palo Alto, CA: Consulting Psychologists Press.

Heppner, P. P., Witty, T. E., \& Dixon, W. A. (2004). Problem-solving appraisal: Helping normal people lead better lives. Counseling Psychology, 32, 466-472.

Holz, N. E., Boecker, R., Jennen-Steinmetz, C., Buchmann, A. F., Blomeyer, D., Baumeister, S., ... Laucht, M. (2016). Positive coping styles and perigenual ACC volume: Two related mechanisms for conferring resilience? Social Cognitive and Affective Neuroscience, 11, 813-820.

Horn, D. I., Yu, C., Steiner, J., Buchmann, J., Kaufmann, J., Osoba, A., ... Walter, M. (2010). Glutamatergic and resting-state functional connectivity correlates of severity in major depression-The role of pregenual anterior cingulate cortex and anterior insula. Frontiers in Systems Neuroscience, 4, 33. https://doi.org/10.3389/fnsys.2010. 00033

Joyce, K. E., Hayasaka, S., \& Laurienti, P. J. (2013). The human functional brain network demonstrates structural and dynamical resilience to targeted attack. PLoS Computational Biology, 9, e1002885.

Karatsoreos, I. N., \& McEwen, B. S. (2011). Psychobiological allostasis: Resistance, resilience and vulnerability. Trends in Cognitive Sciences, 15, 576-584.

Karl, A., Schaefer, M., Malta, L., Dorfel, D., Rohleder, N., \& Werner, A. (2006). A meta-analysis of structural brain abnormalities in PTSD. Neuroscience \& Biobehavioral Reviews, 30, 1004-1031.

Kitano, H. (2004). Biological robustness. Nature Reviews Genetics, 5 , $826-837$.

Kong, F., Hu, S., Wang, X., Song, Y., \& Liu, J. (2015a). Neural correlates of the happy life: The amplitude of spontaneous low frequency fluctuations predicts subjective well-being. NeuroImage, 107, 136-145. https://doi.org/10.1016/j. neuroimage.2014.11.033

Kong, F., Xue, S., \& Wang, X. (2016). Amplitude of low frequency fluctuations during resting state predicts social well-being. Biological Psychology, 118, 161-168.

Kong, F., Wang, X., Hu, S., \& Liu, J. (2015b). Neural correlates of psychological resilience and their relation to life satisfaction in a sample of healthy young adults. NeuroImage, 123, 165-172.

Lazarus, R. S., \& Folkman, S. (1984). Stress, appraisal, and coping. New York, NY: Springer.

Lee, H., Ku, J., Kim, J., Jang, D.-P., Yoon, K. J., Kim, S. I., \& Kim, J.-J. (2014). Aberrant neural responses to social rejection in patients with schizophrenia. Social Neuroscience, 9, 412-423.

Liew, S. L., Santarnecchi, E., Buch, E. R., \& Cohen, L. G. (2014). Noninvasive brain stimulation in neurorehabilitation: Local and distant effects for motor recovery. Frontiers in Human Neuroscience, 8 , 378. https://doi.org/10.3389/fnhum.2014.00378

Litman, J. A. (2006). The COPE inventory: Dimensionality and relationships with approach- and avoidance-motives and positive and negative traits. Personality and Individual Differences, 41, 273-284. https://doi.org/10.1016/j.paid.2005.11.032

Lysaker, P. H., Clements, C. A., Plascak-Hallberg, C. D., Knipscheer, S. J., \& Wright, D. E. (2002). Insight and personal narratives of illness in schizophrenia. Psychiatry: Interpersonal and Biological Processes, 65, 197-206.

McEwen, B. S., Gray, J., \& Nasca, C. (2015). Recognizing resilience: Learning from the effects of stress on the brain. Neurobiology of Stress, 1, 1-11. https://doi.org/10.1016/j.ynstr.2014.09.001

McNally, R. J. (2007). Mechanisms of exposure therapy: How neuroscience can improve psychological treatments for anxiety disorders. Clinical Psychology Review, 27, 750-759. https://doi.org/10.1016/ j.cpr.2007.01.003

Mikulincer, M., \& Solomon, Z. (1989). Causal attribution, coping strategies, and combat-related post-traumatic stress disorder. European Journal of Personality, 3, 269-284.
Mirandola, M., \& Soresi, S. (1991). Contributo all'adattamento italiano del Problem Solving Inventory di Heppner e Petersen. Applied Psychology Bulletin, 198, 9-18.

Nezu, A. M. (1985). Differences in psychological distress between effective and ineffective problem solvers. Journal of Counseling Psychology, 32, 135-138.

Oldfield, R. C. (1971). The assessment and analysis of handedness: The Edinburgh inventory. Neuropsychologia, 9, 97-113.

Ozbay, F., Fitterling, H., Charney, D., \& Southwick, S. (2008). Social support and resilience to stress across the life span: A neurobiologic framework. Current Psychiatry Reports, 10, 304-310.

Parkes, K. R. (1984). Locus of control, cognitive appraisal, and coping in stressful episodes. Journal of Personality and Social Psychology, $46,655-668$

Pascual-Leone, A., Bartres-Faz, D., \& Keenan, J. P. (1999). Transcranial magnetic stimulation: Studying the brain-behaviour relationship by induction of "virtual lesions." Philosophical Transactions of the Royal Society B, 354, 1229-1238.

Pascual-Leone, A., \& Pridmore, H. (1995). Transcranial magnetic stimulation. (TMS). Australian and New Zealand Journal of Psychiatry, 29, 698.

Peterson, A., Thome, J., Frewen, P., \& Lanius, R. A. (2014). Resting-state neuroimaging studies: A new way of identifying differences and similarities among the anxiety disorders? Canadian Journal of Psychiatry, 59, 294-300.

Polania, R., Nitsche, M. A., Korman, C., Batsikadze, G., \& Paulus, W. (2012). The importance of timing in segregated theta phase-coupling for cognitive performance. Current Biology, 22, 1314-1318. https:// doi.org/10.1016/j.cub.2012.05.021

Pu, S., Nakagome, K., Yamada, T., Itakura, M., Yamanashi, T., Yamada, S., ... Kaneko, K. (2016). Social cognition and prefrontal hemodynamic responses during a working memory task in schizophrenia. Scientific Reports, 6, 22500. https://doi.org/10.1038/srep22500

Raichle, M. E. (2015). The brain's default mode network. Annual Review of Neuroscience, 38, 433-447.

Rotter, J. B. (1966). Generalized expectancies for internal versus external control of reinforcement. Psychological Monographs, 80, 1-28.

Santarnecchi, E., Galli, G., Polizzotto, N. R., Rossi, A., \& Rossi, S. (2014). Efficiency of weak brain connections support general cognitive functioning. Human Brain Mapping, 35, 4566-4582. https:// doi.org/10.1002/hbm.22495

Santarnecchi, E., Muller, T., Rossi, S., Sarkar, A., Polizzotto, N. R., Rossi, A., \& Cohen Kadosh, R. (2016). Individual differences and specificity of prefrontal gamma frequency-tACS on fluid intelligence capabilities. Cortex, 75, 33-43.

Santarnecchi, E., Polizzotto, N. R., Godone, M., Giovannelli, F., Feurra, M., Matzen, L., ... Rossi, S. (2013). Frequency-dependent enhancement of fluid intelligence induced by transcranial oscillatory potentials. Current Biology, 23, 1449-1453.

Santarnecchi, E., Rossi, S., \& Rossi, A. (2015). The smarter, the stronger: Intelligence level correlates with brain resilience to systematic insults. Cortex, 64, 293-309. https://doi.org/10.1016/j.cortex.2014.11.005

Scheier, M. F., Weintraub, J. K., \& Carver, C. S. (1986). Coping with stress: Divergent strategies of optimists and pessimists. Journal of Personality and Social Psychology, 51, 1257-1264.

Shen, X., Tokoglu, F., Papademetris, X., \& Constable, R. T. (2013). Groupwise whole-brain parcellation from resting-state fMRI data for network node identification. NeuroImage, 82, 403-415.

Shirer, W. R., Ryali, S., Rykhlevskaia, E., Menon, V., \& Greicius, M. D. (2012). Decoding subject-driven cognitive states with whole-brain connectivity patterns. Cerebral Cortex, 22, 158-165.

Sica, C., Magni, C., Ghisi, M., Altoè, G., Sighinolfi, C., Chiri, L. R., \& Franceschini, S. (2008). Coping Orientation to Problems Experienced-Nuova Versione Italiana (COPE-NVI): Uno strumento per la misura degli stili di coping. Psicoterapia Cognitiva e Comportamentale, 14, 27-53. 
Snowball, A., Tachtsidis, I., Popescu, T., Thompson, J., Delazer, M., Zamarian, L., ... Cohen Kadosh, R. (2013). Long-term enhancement of brain function and cognition using cognitive training and brain stimulation. Current Biology, 23, 987-992. https://doi.org/10. 1016/j.cub.2013.04.045

Southwick, S. M., \& Charney, D. S. (2012). The science of resilience: Implications for the prevention and treatment of depression. Science, $338,79-82$

Sporns, O. (2013). Network attributes for segregation and integration in the human brain. Current Opinion in Neurobiology, 23, 162-171. https://doi.org/10.1016/j.conb.2012.11.015

Sporns, O. (2014). Contributions and challenges for network models in cognitive neuroscience. Nature Neuroscience, 17, 652-660.

Spreng, R. N., \& Schacter, D. L. (2012). Default network modulation and large-scale network interactivity in healthy young and old adults. Cerebral Cortex, 22, 2610-2621.

Spreng, R. N., Sepulcre, J., Turner, G. R., Stevens, W. D., \& Schacter, D. L. (2013). Intrinsic architecture underlying the relations among the default, dorsal attention, and frontoparietal control networks of the human brain. Journal of Cognitive Neuroscience, 25, 74-86. https:// doi.org/10.1162/jocn a 00281

Spreng, R. N., Stevens, W. D., Viviano, J. D., \& Schacter, D. L. (2016). Attenuated anticorrelation between the default and dorsal attention networks with aging: Evidence from task and rest. Neurobiology of Aging, 45, 149-160.

Sprengelmeyer, R., Steele, J. D., Mwangi, B., Kumar, P., Christmas, D., Milders, M., \& Matthews, K. (2011). The insular cortex and the neuroanatomy of major depression. Journal of Affective Disorders, 133, 120-127. https://doi.org/10.1016/j.jad.2011.04.004

Tatti, E., Rossi, S., Innocenti, I., Rossi, A., \& Santarnecchi, E. (2016). Non-invasive brain stimulation of the aging brain: State of the art and future perspectives. Ageing Research Reviews, 29, 66-89.

Thompson, R. W., Arnkoff, D. B., \& Glass, C. R. (2011). Conceptualizing mindfulness and acceptance as components of psychological resilience to trauma. Trauma, Violence \& Abuse, 12, 220-235.

Tryon, W. (2005). Possible mechanisms for why desensitization and exposure therapy work. Clinical Psychology Review, 25, 67-95.

Tsai, J., Harpaz-Rotem, I., Pietrzak, R. H., \& Southwick, S. M. (2012). The role of coping, resilience, and social support in mediating the relation between PTSD and social functioning in veterans returning from Iraq and Afghanistan. Psychiatry: Interpersonal and Biological Processes, 75, 135-149.

Uddin, L. Q. (2014). Salience processing and insular cortical function and dysfunction. Nature Reviews Neuroscience, 16, 55-61.

van der Werff, S. J. A., van den Berg, S. M., Pannekoek, J. N., Elzinga, B. M., \& van der Wee, N. J. A. (2013). Neuroimaging resilience to stress: A review. Frontiers in Behavioral Neuroscience, 7, 39. https://doi.org/10.3389/fnbeh.2013.00039

van Tol, M.-J., van der Wee, N. J. A., van den Heuvel, O. A., Nielen, M. M. A., Demenescu, L. R., Aleman, A., ... Veltman, D. J. (2010). Regional brain volume in depression and anxiety disorders. Archives of General Psychiatry, 67, 1002.

Wang, L., Song, M., Jiang, T., Zhang, Y., \& Yu, C. (2011). Regional homogeneity of the resting-state brain activity correlates with individual intelligence. Neuroscience Letters, 488, 275-278.

Waugh, C. E., Thompson, R. J., \& Gotlib, I. H. (2011). Flexible emotional responsiveness in trait resilience. Emotion, 11, 1059-1067.

Witek-Janusek, L., Albuquerque, K., Chroniak, K. R., Chroniak, C., Durazo-Arvizu, R., \& Mathews, H. L. (2008). Effect of mindfulness based stress reduction on immune function, quality of life and coping in women newly diagnosed with early stage breast cancer. Brain, Behavior, and Immunity, 22, 969-981. https://doi.org/10.1016/j.bbi. 2008.01.012

Wolters Gregório, G., Ponds, R. W. H. M., Smeets, S. M. J., Jonker, F., Pouwels, C. G. J. G., Verhey, F. R., \& van Heugten, C. M. (2015). Associations between executive functioning, coping, and psychosocial functioning after acquired brain injury. British Journal of Clinical Psychology, 54, 291-306.

Zalesky, A., Cocchi, L., Fornito, A., Murray, M. M., \& Bullmore, E. (2012). Connectivity differences in brain networks. NeuroImage, 60, 1055-1062. https://doi.org/10.1016/j.neuroimage.2012.01.068

Zhang, D., \& Raichle, M. E. (2010). Disease and the brain's dark energy. Nature Reviews Neurology, 6, 15-28.

Zhou, J., Greicius, M. D., Gennatas, E. D., Growdon, M. E., Jang, J. Y., Rabinovici, G. D., ... Seeley, W. W. (2010). Divergent network connectivity changes in behavioural variant frontotemporal dementia and Alzheimer's disease. Brain, 133, 1352-1367. https://doi.org/ 10.1093/brain/awq075 